\title{
Rag Hamsadvani Relieves Fatigue and Elevates The Mood of Listeners \\ A Case Study From The Music Medicine Unit, MGMCRI \\ P.Barathi *
}

Mr. S, a 46-year-old, married male with persistent fatigue, muscle pain, and insomnia was referred to the Music Medicine Unit. To identify the cause of his fatigue, routine blood test along with thyroid, liver, and renal functions were performed. However, nothing abnormal was detected. Additional laboratory evaluations were also carried out to rule out systemic infections. The fatigue persisted for about 6 months, could not be relieved by rest and a concrete medical diagnosis could not be arrived at. Because he had only 2 accompanying symptoms (sleep dysfunction and musculoskeletal pain), he did not meet all of the criteria for Chronic Fatigue Syndrome (CFS) and his fatigue was diagnosed as Idiopathic Fatigue Syndrome (ICF).

The Piper Fatigue Scale (PFS) ${ }^{1}$ was used to assess the severity of his fatigue. The PFS is composed of 22 numerically scaled, " 0 " to " 10 " items that measure four dimensions of subjective fatigue: behavioral/severity (6 items); affective meaning ( 5 items); sensory ( 5 items); and cognitive/mood (6 items). These 22 items are used to calculate the four sub-scale/dimensional scores and the total fatigue scores. The severity codes range from "0" or "none", 1-3 (mild), 4-6 (moderate) to a score of 7-10, which is designated as severe in intensity.

Rag Hamsadvani was administered through head phones for 40 min, everyday in the morning at 10 am, for a duration of 1 month. To facilitate continuous listening, he was given the music CD to listen at home, whenever he was unable to come to the MMU for listening to the music. After 1 month of continuous listening, his fatigue score came down from 7 (severe) to 4 (moderate). There were also considerable changes in his lifestyle. Before the therapy he stated, "I never feel refreshed after waking up in the morning", "I am not able to remember things properly", "In the daytime, I am constantly feeling the desire to sleep". After 4 weeks of listening to Rag Hamsadvani, he no longer complained of severe fatigue, and his family noticed that he was much more lively and energetic than before.

\section{ABout RAg HaMSADVANI}

Rag Hamsadvani radiates positive energy imbibed with vitality, enthusiasm and optimism. Sri Muthuswamy Dikshitar, one of the trinities of South Indian Carnatic music, has composed a kriti in Hamsadvani, 'Vatapi ganapathim', which is played at the beginning of musical concerts to enliven the audience. In this composition, he glorifies Lord Ganesha as destroying all sadness/obstacles. It is interesting to note that most of the compositions in Hamsadvani by various composers is based on an invocation to Lord Ganesha, who is the Lord responsible for clearing all obstacles and hindrances, and is therefore invoked for auspiciousness at the beginning of any event.

The nature of emotion evoked on listening to Rag Hamsadvani- joy, happiness, enthusiasm.

Hamsadhvani literally means 'Sound of Swans'. It has a pentatonic scale, which is a derived scale, as it does not have all the seven musical notes.

Hamsadhvani does not contain Ma (madhyamam) or Dha (dhaivatam).

ārohana or ascent: S R2 G3 P N3 S

avarohana or descent: S N3 P G3 R2 S

This rag instills joy and happiness in the mind of listeners.

\section{OTHER FACTORS RESPONSIBLE FOR RELIEF FROM FATIGUE}

1) Certain elements within the music, such as a change of melodic line or rhythm, that create expectations about

\footnotetext{
* Dr.P.Barathi, M.D.,

Prof of Physiology, Music Therapist

e-mail : iyer.bharthi@gmail.com
} 
the future development of the music. The expectation the listener has about the further course of musical events is a determinant for the experience of 'musical emotions. Music arouses expectations, which may or not be directly and immediately satisfied. The greater the build-up of suspense of tension, the greater the emotional release upon resolution ${ }^{2}$. The primary dimension on which emotion is experienced is in terms of a build-up of tension and release of tension, both within the music and in the listener. The more elaborate the build-up of tension to a certain climax, the more intense emotions will be experienced. When resolution follows, relaxation follows.

2) A 'naive' listener without much music theoretical knowledge has a predominant affective response towards music whereas a trained listener reacts mostly cognitively while listening to music ${ }^{3}$.

3) Both Berlyne ${ }^{4}$, Mandler ${ }^{5}$ see arousal as an important factor in emotional experiences while listening to music. A typical example of physical experiences reported when listening to music is the experience of thrills. Goldstein ${ }^{6}$ illustrated that thrills were experienced by $96 \%$ of the subjects. Such a thrill can be experienced, when Hamsadvani is played, accompanied by a fast rhythm. This may be responsible for the mood elevation induced on listening to this rag.

To conclude, Rag Hamsadvani may have a potential positive effect on ICF, and it can be used as an alternative treatment of choice for patients with ICF. Well-designed clinical trials including randomized controlled trials with large number of participants are necessary to assess the exact mechanisms involved.

\section{Other BENEFITS OF LISTENING TO RAg HAMSADVANI}

Mr. Y was referred to the Music Medicine Unit with the complaints of extreme anxiety, palpitations and dyspnea, after her son met with a brain injury in a road traffic accident. She was found to have a normal ECG, and her lung function tests did not reveal any abnormality. She was diagnosed as suffering from anxiety due to her son's poor prognosis.

She was administered Rag Hamsadvani at a speed of $42 / \mathrm{mt}$ (the speed of the patient's breath rate) for a duration of $15 \mathrm{mts}$, followed by the same Rag at a speed of $12 / \mathrm{mt}$. Her breath rate gradually subsided to a count of $16 / \mathrm{mt}$.

\section{THE ROLE OF ENTRAINMENT IN MUSIC THERAPY:}

Entrainment is one thing time locking into another7. In the 1700's German scientists, working with grandfather clocks in their workspace, left one night after setting the clocks, noticing all the clock's pendulums were swinging randomly. The next morning they found that all of the pendulums were swinging together, again, one thing time locking into another. Entrainment is a natural phenomenon of physics. With this idea of entrainment, the music therapist matches, for example, the music's tempo to the patient's breath rate (heart rate or mood can also be considered). Entrainment is complete when the music therapist can affect change in the patient's physiology through musical variation. Example: If a painful patient is anxious and breathing rapidly, the music therapist will play (live) music at the speed (tempo) that matches the patient's rapid breath rate.

When it is noticed that the patient is focused on the music, the music therapist will gradually begin to slow the music down, thereby effectively slowing the patient's breath rate at the same time. This completes the entrainment process. Entrainment is also very effective for pain management.

In an entrainment study Rider used different types of music and imagery to reduce pain and effect muscle relaxation. The entrainment music, which was one of five conditions in which the prevalent mood shifted from tension to relaxation, was significantly the most effective condition in reducing pain and EMG levels. One key to music's well-documented positive effects on pain management and entrainment is preferred music.

Through assessment, the music therapist will help the patient (or family) identify music that will elicit positive, euphoric or sedative responses. Or very simply, what music does the patient have the most positive associations? When we can identify music that elicits feelings and emotions of past positive experiences, the patient can use these conditioned responses to override feelings of anxiety and pain.

Because of its pentatonic structure and non-complicated ascent and descent, this rag can be ideally used for entrainment purposes. 


\section{ReferenCES}

1. Piper BF, Dibble SL, Dodd MJ, Weiss MC, Slaughter RE, Paul SM. (1998). The revised Piper Fatigue Scale: psychometric evaluation in women with breast cancer. Oncol Nurs Forum ;25(4): 677-84.

2. Meyer, Leonard B. (1956). Emotion and meaning in music. Chicago: Chicago Press.

3. Jansma, Mireille \& Vries, Bart de (1995). Muziek en emotie (pp. 204-222). In: Evers, Jansma, Mak, De Vries (Eds). Muziekpsychologie. Assen: Van Gorcum.

4. Berlyne, Daniel Ellis (1971). Aesthetics and psychobiology. New York: Appleton Century-Crofts.

5. Mandler, George (1984). Mind and body: Psychology of emotions and stress. New York: Norton.

6. Goldstein, Avram (1980). Thrills in response to music and other stimuli. Physiological Psychology, 8, 1, 126-129.

7. Rider, M.S., Floyd, J.W., \& Kirkpatric, J. (1985). The effect of music, imagery, and relaxation on corticosteroids and the re- entrainment of circadian rhythms. Journal of Music Therapy, 22(1), 46-58. 